\title{
Des « peuples du bétail »
}

Origines mythiques et pratiques rituelles de l'Élevage en Afrique de l'Est

\section{Pierre Bonte}

\section{CpenEdition}

\section{Journals}

Édition électronique

URL : https://journals.openedition.org/tc/1116

DOI : $10.4000 /$ tc. 1116

ISSN : 1952-420X

Éditeur

Éditions de l'EHESS

\section{Édition imprimée}

Date de publication : 1 décembre 2004

ISSN : 0248-6016

\section{Référence électronique}

Pierre Bonte, «Des « peuples du bétail » », Techniques \& Culture [En ligne], 43-44 | 2004, mis en ligne le 15 avril 2007, consulté le 29 septembre 2022. URL : http://journals.openedition.org/tc/1116 ; DOI : https://doi.org/10.4000/tc.1116

Ce document a été généré automatiquement le 29 septembre 2022.

Tous droits réservés 


\title{
Des « peuples du bétail »
}

\author{
Origines mythiques et pratiques rituelles de l'Élevage en Afrique de l'Est
}

\author{
Pierre Bonte
}

1 Les Nuer sont sans nul doute l'une des populations au monde les mieux connues des anthropologues grâce à la remarquable somme monographique, dispersée à travers un certain nombre d'ouvrages, que leur a consacrée Edward E. Evans-Pritchard. Dans le principal et le mieux connu de ses ouvrages (1968), il les définit comme un «peuple du bétail»(cattle people). Définition curieuse car, si les Nuer sont effectivement des éleveurs de bovins et d'autres sortes de bétail, leur mode de vie dépend tout autant de l'agriculture et de la pêche que de l'élevage.

2 La notation ne prend sens que si l'on rapproche le statut symbolique et social du bétail -plus particulièrement des bovins- dans la société nuer de celui qu'il occupe dans la majorité des sociétés est-africaines, qu'elles soient spécialisées -l'exemple le plus connu est celui des Masai- ou non dans les tâches pastorales. Moins de deux décennies avant qu'Evans-Pritchard ne rédige son premier ouvrage sur les Nuer, d'autres anthropologues avaient relevé ce trait culturel propre aux sociétés est-africaines, et parlé d'un « complexe du bétail » (cattle complex) pour désigner la relation particulière que ces populations entretiennent avec leurs bovins (Herskovits 1926), voire de «boomanie » ou de "boolâtrie " pour souligner que ce rapport a moins à voir avec la dimension matérielle de l'élevage, qui parfois ne joue qu'un rôle second dans leurs modes de production et de vie, qu'avec la manière dont le bétail s'inscrit dans les représentations symboliques et religieuses.

Techniques et cultures pastorales

3 Les données archéologiques attestent d'une spécialisation pastorale de ces populations est-africaines depuis le IIIème millénaire av. J.-C. L'évolution des pratiques d'élevage, du fait de la diffusion ultérieure du zébu en particulier, relève ainsi d'une histoire commune, faite de migrations de "peuples d'éleveurs", de la construction d'hégémonies locales, de la spécialisation à des degrés divers de ces populations dans l'élevage en fonction de conjonctures historiques et écologiques (Galaty \& Bonte 1991), etc., histoire qui se manifeste aussi par une relative permanence des techniques d'élevage -que l'on retrouve sans grandes différences dans toute l'aire concernée ${ }^{1}$. 
Il serait donc difficile d'introduire, en se référant à ces techniques pastorales largement identiques, un ordre qui permette de classer les diverses populations de cette aire culturelle en fonction de leurs pratiques de l'élevage. Les différences que l'on peut relever tiennent à la place de l'Élevage ${ }^{2}$ en tant qu'ensemble de croyances, de savoirs et de pratiques, d'ordre technique certes, mais aussi rituel, social, économique..., associés exclusivement à la possession et à l'utilisation des bovins ${ }^{3}$.

En fait, peu de sociétés est-africaines sont spécialisées dans l'élevage des bovins; les Masai du Kenya et de la Tanzanie représentent l'une des rares exceptions. Il n'en est que plus remarquable de constater que même dans ces sociétés, l'Élevage est une activité réservée aux hommes, les femmes étant exclues de la possession du bétail et de la majorité des tâches le concernant. Chez les Masai comme dans les sociétés agropastorales voisines, elles sont associées à l'Agriculture -la majuscule souligne là aussi que sous ce terme qui renvoie à une organisation technique de la production, se révèlent des croyances, des savoirs et des pratiques qui ne peuvent être appréciés en fonction des seuls référents matériels ${ }^{4}$. Mais sur le plan de l'organisation régionale de la production, l'affaire apparaît plus complexe encore. Entre pasteurs spécialisés et agropasteurs voisins, cette distinction inspirée des valeurs de genre -le masculin pastoral et le féminin agricole- contribue aussi à l'établissement de hiérarchies qui prennent alors un tour «ethnique ». De même, les relations entre les éleveurs et les chasseurscollecteurs (Torrobo) qui vivent dans l'interstice des sociétés pastorales et agropastorales locales, s'établissent en termes de hiérarchies qui renvoient aux valeurs dominantes masculines de ceux qui, pratiquant l'Élevage, se trouvent en position d'incarner la société dans son ensemble. Pour comprendre ces hiérarchies, et l'organisation, qui en découle, des techniques de production pastorale, il faut nous pencher sur les représentations que se font l'ensemble de ces populations des origines du bétail et des fondements de l'Élevage.

L'origine cosmique du bétail

6 Dans diverses sociétés, les récits qui rendent compte des origines du monde (cosmogenèse) et de l'humanité (anthropogenèse) ont pour trait commun d'associer le bétail au monde surnaturel et de justifier ainsi la relation qu'il entretient depuis ce temps des origines avec les groupes qui pratiquent l'Élevage.

7 Des mythes de cet ordre ont été recueillis chez les Masai par divers auteurs. L'un des plus répandus est celui de "L'escalade de la falaise » (Galaty 1977). L'humanité vivait initialement dans un cratère désolé et sec, entouré de hautes falaises. Il n'existait à cette époque aucune différence d'âge, de parenté ou de langue entre les hommes, et tous possédaient du bétail. Un jour, l'un d'eux découvrit des pâturages verdoyants audelà des falaises et ils décidèrent d'y mener leurs vaches. Ils construisirent un pont pour pouvoir franchir la falaise avec leur bétail. Une fois traversé par les vaches et une partie des hommes, le pont s'écroula. Les Masai sont les Éleveurs qui purent sortir du cratère avec le bétail; ceux qui y restèrent sans bétail sont les ancêtres des Il Meek, des Agri-culteurs. Ce mythe, comme d'autres récits, souvent organisés autour du thème de l'ascension, de la mise à distance, justifie la distinction, d'ordre hiérarchique dans les représentations propres aux uns et aux autres, entre les Masai, qui vivent en priorité de l'élevage, et leurs voisins. Un autre mythe rapporte que le bétail fut donné aux hommes par la divinité suprême après qu'ils eurent été chassés d'une sorte de paradis originel, également caractérisé par l'indifférenciation des genres, des états et des statuts. Les vaches descendirent du ciel le long d'une corde, mais celle-ci cassa avant que tous les 
hommes n'aient reçu une part de bétail. Il en résulta deux catégories d'humains : ceux qui s'occupent d'Élevage et ceux qui pratiquent l'Agriculture ${ }^{5}$.

8 La distance entre Élevage et Agriculture est parfois soulignée en termes moins topiques qu'éthiques. C'est le thème d'un autre mythe d'origine masai. L'ancêtre commun des hommes, appelé Le-eyo, terme générique pour l'homme, avait reçu le bétail d'une divinité. Il avait deux fils auxquels il proposa de choisir leur part d'héritage. L'aîné, qui sera l'ancêtre des agriculteurs Il Meek, lui demanda une part de tous les biens qu'il possédait. Le cadet, ancêtre des Masai, se contenta de réclamer le chasse-mouche de son père, emblème de l'autorité masculine et du prestige des Anciens dans une société organisée au sein d'un système rigide de classes d'âge. Il reçut la bénédiction de son père et la totalité du bétail, ainsi associé aux valeurs masculines incarnant l'autorité et l'ordre social. Car le bétail est masculin et les femmes en furent exclues dès les origines. D'autres récits d'origine le rappellent de manière insistante.

9 Certes, une relative indifférenciation est généralement posée dans les temps primordiaux. Dans un mythe rapporté par Merker (1971), aux origines, les hommes possédaient les vaches et les femmes étaient propriétaires des animaux «sauvages " qui vivaient en symbiose avec les humains. Mais un jour, une femme tua l'un de ces animaux et ceux-ci décidèrent alors de gagner seuls désormais les pâturages. Les hommes restèrent ainsi les possesseurs exclusifs des animaux domestiques. Un autre mythe, rapporté par le même auteur, explique aussi l'exclusion des femmes et il nous fournit simultanément une clef de l'origine du sacrifice.

10 Les hommes, chassés du paradis primordial, reçurent le bétail de Dieu dans les conditions précédemment exposées. Dieu s'aperçut que les hommes ne pouvaient se nourrir uniquement du lait des vaches qu'il leur avait accordées; il leur permit alors de prélever le sang des animaux vivants et leur fournit à cette fin l'arc et la flèche ${ }^{6}$, leur interdisant cependant formellement de mettre à mort les animaux. Mais un jour, un homme, si pauvre qu'il ne possédait que quelques vaches et vivait des produits de la cueillette, vit son fils tomber malade. Pour guérir l'enfant, son épouse lui demanda de prélever du sang sur l'une de ses vaches. Il le fit, mais la quantité de sang ne fut pas suffisante et l'état de l'enfant empira. Sa femme lui demanda alors de tuer l'une de ses bêtes pour guérir l'enfant en lui donnant de la viande. L'homme refusa, mais durant son absence, la mère, pour sauver son fils, tua l'une des bêtes du troupeau. Dieu chassa alors tous les habitants du campement qu'il brûla; la femme et l'enfant, qui s'étaient attardés, périrent dans l'incendie. Depuis, seules les femmes «travaillent ", c'est-à-dire pratiquent l'Agriculture conçue, contrairement à l'Élevage, comme une peine ${ }^{7}$. Conscient des difficultés des hommes, Dieu les autorisa à consommer la chair de leurs animaux à condition qu'ils soient sacrifiés rituellement. Les femmes, leçon ultime du mythe, ne peuvent pratiquer l'Élevage ni posséder le bétail ${ }^{8}$ parce qu'elles ont manifesté leur incapacité à en faire un "bon usage", en l'occurrence en tuant les animaux qui leur étaient confiés à des fins personnelles, celles du bien-être de leurs enfants, et non selon les règles surnaturelles qui avaient présidé à leur attribution à l'humanité.

Spécialisation pastorale et place de l'agriculture

11 Les Masai, dont nous venons de rapporter les mythes d'origine, font partie des rares sociétés qui présentent, en Afrique de l'Est, une forte spécialisation pastorale impliquant des relations suivies avec les sociétés agricoles voisines pour obtenir des compléments de nourriture céréalière; on pourrait en trouver l'équivalent chez 
certains Peuls d'Afrique occidentale. Ces sociétés de cultivateurs partagent pour une part les valeurs pastorales masculines que nous venons d'évoquer, et qui sont largement répandues sur tout le continent africain. Il n'en est que plus intéressant d'analyser les variations de cette idéologie pastorale observées dans les sociétés agropastorales proches, qui sont amenées à concilier les valeurs pastorales masculines dominantes avec l'implication, fortement affirmée, des valeurs féminines dans l'Agriculture. Nous examinerons dans cette perspective un mythe d'origine des Gogo de Tanzanie, voisins des Masai, chez lesquels l'agriculture est incontestablement l'activité principale; ils se réfèrent pourtant aux mêmes valeurs pastorales et leurs mythes nous rapportent dans des termes comparables, mais aussi profondément divergents de par la place qu'ils accordent aux médiations féminines, l'origine de leur bétail (Rigby 1971).

Un jour, les hommes rejetèrent hors de la société des origines une femme divorcée avec ses enfants. Protégée par le lion, elle se réfugia dans la nature où elle put élever ses enfants, mais trompée par la hyène, elle se trouva aussi rejetée du monde sauvage. Seul le zèbre -animal sauvage considéré comme étant le plus proche de l'homme- accepta de la protéger et il finit par l'emporter sur le lion. La femme et les enfants purent alors réintégrer l'humanité en amenant avec eux les bovins, jusque là sauvages.

Ce sont donc les femmes ici qui apportent le bétail aux hommes et qui jouent un rôle de médiation non sans ambiguïté, comme l'attestent les rôles assumés dans le mythe : la femme est divorcée, la hyène est un animal hermaphrodite dans les mythes africains, le zèbre se situe entre le monde sauvage et le monde humain. On retrouve la même ambiguité dans un autre mythe d'origine du bétail et de la vie sociale, recueilli cette fois chez les Atuot, voisins et proches des Nuer dont ils partagent les valeurs pastorales (Burton 1981).

14 Aux temps primordiaux, les hommes vivaient dans la forêt avec des buffles sauvages, sans contact avec les femmes qui demeuraient près de la rivière, dans un autre campement, pêchant, cultivant et détenant les vaches. Les vagins des femmes étaient alors fermés et elles concevaient - des filles exclusivement- en se baignant dans les eaux de la rivière. Un buffle disparut un jour du campement des hommes et l'un d'entre eux suivit sa trace jusqu'au campement des femmes. Elles ne purent l'informer du sort de l'animal, mais elles découvrirent le pénis, et l'homme le vagin. Les femmes se précipitèrent sur l'homme, qui en mourut, mais hommes et femmes se rapprochèrent. Les hommes s'aperçurent alors que les femmes s'intéressaient surtout à l'agriculture et ils leur volèrent les vaches après avoir chassé les buffles dans la forêt. Chaque femme choisit alors un homme et les hommes exigèrent de se marier. L'aînée des femmes demanda qu'ils donnent des vaches en échange, sous forme de prestation matrimoniale.

15 Ce sont encore les femmes qui apportent les vaches à l'humanité, mais elles s'en désintéressent, et les hommes se les approprient pour se marier et pour contrôler la vie sociale. Si les valeurs pastorales restent dominantes, s'esquissent cependant, dans ces sociétés du sud du Soudan dont font partie les Nuer, un ensemble de valeurs complémentaires associées au monde féminin, à l'agriculture, à l'eau et à la pêche, ainsi qu'à la fécondité. Ces représentations jouent un rôle décisif pour comprendre les différences que présentent ces sociétés, atuot, dinka, nuer, partageant un passé commun qui remonte à quatre ou cinq siècles, non seulement en ce qui concerne les valeurs auxquelles elles se réfèrent, mais dans leur organisation sociale ${ }^{9}$.

Les Nuer, « un peuple du bétail»? 
Qu'est-ce donc qui justifie le fait qu'Evans-Pritchard réserve cette appellation aux seuls Nuer, dont le mode de vie est en partie le même que celui de leurs voisins atuot et dinka, qui associent à l'élevage l'agriculture et la pêche, et reconnaissent par ailleurs les mêmes valeurs pastorales? Parallèlement à ces valeurs pastorales, propres aux hommes, se dessine un autre ensemble de repré-sentations, liées à l'eau, aux rivières et aux lacs, à la pêche plus encore qu'à l'agriculture, qui apparaissent comme des valeurs féminines. Nous les avons déjà repérées dans le mythe atuot. Selon un autre mythe des Atuot, leur territoire ancestral était initialement sec et désolé. Un seul puits était disponible mais il ne fournissait que du sang. On y noya une belle jeune fille et ce puits devint le lac Nyirol où les Atuot trouvèrent une pirogue, un harpon et les différentes espèces de poissons. Si l'on examine l'organisation sociale de ces peuples -les Nuer exceptés-, on s'aperçoit que de telles représentations définissent des valeurs féminines mais aussi des valeurs d'autorité.

17 Chez les Atuot et chez les Dinka, les offices politico-rituels sont exercés dans un contexte de filiation cognatique, par les "maîtres du harpon» qui transmettent héréditairement un pouvoir d'origine divine. Ils assument une fonction assez semblable à celle des " prêtres de la terre » chez les Nuer, plus souvent appelés « chefs à la peau de léopard $»^{10}$. Cette similitude met en évidence une opposition valorisée entre le harpon, la pêche et la parenté cognatique d'une part, et d'autre part, entre la lance, la guerre et la parenté agnatique, ainsi que l'a montré Evans-Pritchard dans le long chapitre de Nuer religion (1956) consacré au symbolisme de la lance.

On a longtemps pensé que les particularités des Nuer, qui les distinguaient de leur voisins, tenaient à la mise en place du système lignager patrilinéaire et segmentaire qu'Evans-Pritchard a décrit comme la forme paradigmatique des sociétés " acéphales ", système qui aurait facilité l'expansion de ces Nuer aux dépens des autres (Sahlins 1961). Il est vrai que les valeurs patrilinéaires, masculines (lance) et pastorales, s'inscrivent au cœur des représentations nuer. Cependant, les distinguent-elles significativement des Dinka, par exemple? Peu à peu, cette idée a été remise en question par les anthropologues. Constatant que les groupes de parenté sont souvent organisés selon une filiation agnatique, certains d'entre eux ont parlé d'« illusion agnatique » (Southall 1985) et reconsidéré la nature de l'organisation lignagère segmentaire décrite par Evans-Pritchard. "D'où sont venus tous ces lignages?", s'interroge pour sa part Verdon (1984), qui souligne que l'ultime cohérence de l'ordre patrilinéaire, masculin et pastoral de la société nuer relève d'une lecture de la structure sociale mettant en évidence les valeurs hiérarchiques de l'Élevage et les représentations qui les fondent sur le bon usage des vaches. Les lignages sont des groupes constitués à des fins rituelles -et le bon usage rituel du bétail est de nature sacrificielle, nous y reviendrons plus longuement. Les lignages désignés comme «minimaux " par Evans-Pritchard sont en fait, selon Verdon, des "groupes rituels patrifiliaux» rassemblés pour l'utilisation sacrificielle des bovins. Les relations agnatiques plus larges s'expliquent non plus par l'usage sacrificiel du bétail vivant mais par l'idée d'un troupeau détenu en commun aux origines, qui crée entre les hommes des liens ancestraux.

En définitive, et là Evans-Pritchard ne s'y était pas trompé, c'est en tant que « peuple du bétail » que les Nuer ont créé leur identité et produit l'ordre social original qui a retenu l'attention des anthropologues. "Les Nuer ne sont Nuer que lorsqu'ils se réunissent pour garder du bétail nuer » (Burton $1981: 570$ ). C'est en développant certaines valeurs liées au bétail, telles que les révèlent les mythes d'origine et qu'elles se traduisent dans 
les rituels, sacrificiels en particulier, que s'est forgée et étendue territorialement cette société, et que les Nuer ont été amenés, par la même occasion, sans que leur mode de vie ne s'en distingue radicalement sur le plan matériel, à produire plus de bétail que leurs voisins dinka. Je me suis attaché à montrer ailleurs que la spécialisation pastorale de certaines de ces sociétés d'Afrique de l'Est s'expliquait moins par des raisons tenant à l'aridité des milieux où à des contraintes techniques que par les effets de ces valeurs pastorales ${ }^{11}$.

Il est à noter que les Dinka vivent aussi dans un univers de représentations structuré par ces valeurs pastorales - dans ce que j'appellerai désormais un « univers du bétail ». L'anthropologue britannique qui a mené les premiers travaux sur les Dinka, Godfrey Lienhardt, relève ainsi que le terme qu'ils emploient pour désigner l'unité territoriale -la «tribu», wut- dénote initialement le campement de bétail. Il observe que l'arrangement, l'organisation et la segmentation de ces campements de bétail constituent le paradigme selon lequel s'organise la répartition des membres de la tribu en groupes de filiation commune. Les structures sociales des Nuer et des Dinka ne sont pas foncièrement différentes. Certes, les Dinka s'organisent en lignages et en tribus rattachés par des liens maternels, mais chez les Nuer, de nombreux lignages ont des ancêtres féminines et s'inscrivent dans la lecture agnatique de l'ordre social comme des « enfants des filles » des lignages localement dominants.

21 L'incise, sans doute bien trop rapide, que nous venons de consacrer aux sociétés nilotiques soudanaises sur lesquelles l'œuvre pionnière d'Evans-Pritchard a, à juste titre, attiré l'attention -suscitant une abondante littérature dont nous avons pu bénéficier-, fait apparaître les logiques classificatoires qui sont mises en jeu dans le cadre de cet « univers du bétail », commandé par les valeurs dominantes de l'Élevage et l'idée d'une origine surnaturelle des bovins. Le bétail, les bovins plus précisément -car le petit bétail, ovins et caprins, qui sont détenus aussi en grand nombre dans ces sociétés est-africaines, n'est pas porteur des mêmes valeurs-, joue un rôle décisif pour distinguer, pour classer les hommes et les femmes, les éleveurs et les agriculteurs, comme les peuples en général. Plus globalement, la référence aux valeurs dominantes du bétail intervient pour définir la place de l'ensemble des activités techniques. Revenant aux sociétés masai, nous allons le mettre en évidence à propos de la place qu'occupent en leur sein un certain nombre de populations qui vivent de la chasse et de la collecte.

Masai et Torrobo : l'établissement des hiérarchies

Les Torrobo sont des chasseurs-collecteurs qui vivent en bandes dispersées parmi les Masai. Leurs contacts avec ceux-ci sont assez étroits pour qu'ils partagent certaines de leurs valeurs pastorales, en fonction desquelles vont s'organiser leurs relations économiques et sociales ${ }^{12}$. En témoigne le récit mythique qui explique l'origine de la distinction entre les Masai et les Torrobo.

En créant le monde, Dieu trouva trois êtres : un Torrobo, un éléphant et un serpent. Il donna une vache au Torrobo. Celui-ci tua le serpent qui le menaçait, puis l'éléphant qui troublait l'abreuvoir de la vache. Le fils de l'éléphant partit dans un autre pays où il rencontra un Masai qui, ayant appris l'histoire, partit à la recherche du Torrobo. Entretemps, Dieu avait demandé au Torrobo de construire un campement et de sacrifier un veau pour qu'il lui accorde sa bénédiction et du bétail. Par ruse, le Masai réussit à recevoir de Dieu le bétail destiné au Torrobo et il en devint dès lors le propriétaire exclusif. 
Dautres mythes (Galaty 1977) se construisent sur un schéma identique. Les Torrobo sont les premiers détenteurs du bétail et bénéficient de la bénédiction divine, qu'ils perdent par suite d'une négligence ou en raison de défauts qui leur sont propres : il s'agit souvent de la gloutonnerie qui leur est attribuée de manière stéréotypée. John Galaty rapproche certains de ces récits de ceux qui justifient l'exclusion des femmes de la possession du bétail parce qu'elles n'ont pas hésité à tuer un animal pour nourrir leur enfant, par exemple. De manière générale, ces mythes d'origine du monde, de l'humanité et du bétail s'organisent selon des principes comparables.

Ils se réfèrent très généralement à une entité surnaturelle toute-puissante et créatrice, mais qu'on ne peut évoquer qu'à travers ses créations, y compris celle de multiples êtres surnaturels plus proches des hommes, et le mode de communication qui s'établit entre elle et les hommes par l'intermédiaire du bétail, don et bénédiction de cette entité. Une indifférenciation primordiale s'inscrit dans l'ordre de la création, et paraît même parfois la précéder tant les représentations sont floues en ce domaine. Il ne s'agit pas d'un univers chaotique mais d'un monde qu'ordonnent d'autres règles que celles qui gouvernent la société actuelle et dont elles constituent parfois une inversion, sinon une transgression. Les femmes et les chasseurs-collecteurs torrobo peuvent détenir le bétail, ou encore celui-ci est attribué à l'ensemble de l'humanité; les animaux sauvages et domestiques ne sont pas distingués; les peuples, les langues, les groupes de parenté ou d'âge ne sont pas différenciés; la distinction des sexes même peut rester virtuelle, etc. Bref, les activités humaines ne sont pas dotées d'un caractère social mais portent en elles tous les traits de la société qui va naître de cet état des origines.

L'opérateur des différenciations est le bétail, en l'occurrence les bovins qui font partie de la création divine, qui établissent, et rétabliront à tout moment à travers le sacrifice, un lien fondamental entre transcendance et immanence. La gloutonnerie ou tout simplement la faim distinguent les hommes des femmes, les Masai des agriculteurs Il Meek. Le bétail est approprié par ruse, quelquefois aux dépens de la divinité, trompée dans ses intentions premières. Le cadet se révèle plus intelligent que l'aîné. Les hommes dérobent le bétail aux femmes, etc. Alors même que les catégorisations sociales fondées dans les récits mythiques peuvent apparaître figées, le foisonnement de ces récits révèle les possibilités de leur manipulation, que l'on observe par ailleurs à travers l'histoire propre de ces sociétés : en développant les valeurs pastorales et en s'identifiant comme un "peuple du bétail », les Nuer se distinguent des Dinka puis les refoulent hors d'un vaste territoire. En acquérant du bétail, les Torrobo deviennent des Masai, et en le perdant ceux-ci se font Torrobo. Certaines sociétés se spécialisent dans l'élevage des bovins. Des hégémonies locales s'établissent puis s'effondrent.

Ainsi entendu, l'Élevage doit être conçu comme un ensemble de pratiques, de savoirs et de représentations concernant les bovins, dans le contexte desquels il est impossible de distinguer des déterminations purement techniques, pas plus que des déterminations strictement écologiques, car la mise en œuvre des pratiques ou des savoirs techniques (Bonte 1985) ou encore les contraintes du milieu (Bonte 1981), ne peuvent être analysées indépendamment des représentations qui commandent l'exercice de cet Élevage. Ce sont même au contraire celles-ci qui donnent sa signification première à l'organisation matérielle et sociale de ces populations. Elles justifient la répartition des activités techniques entre hommes et femmes, chasseurs, agriculteurs et éleveurs, mais aussi entre éleveurs, forgerons -qui jouent un rôle essentiel dans ces sociétés- et 
spécialistes des services rituels ${ }^{13}$ qui contribuent impérativement au bien-être du bétail et des hommes (Becquemont \& Bonte 2004, s/presse).

On pourrait être tenté de reprendre, pour désigner, dans ces sociétés est-africaines, les relations entre les différents groupes définis comme engagés dans l'Élevage, l'Agriculture, la Chasse-cueillette, la Forge et la Spécialisation rituelle, la notion de hiérarchie introduite par Louis Dumont (1983). Dans un esprit proche de celui qui préside à la présente étude, Dumont envisage cette notion de hiérarchie comme expression de l'inscription des activités sociales dans une totalité qui leur donne sens. $\mathrm{Au}$ sein des sociétés holistes -que Dumont oppose aux formes modernes de l'individualisme-, pareilles activités obéissent à l'affirmation de valeurs qui organisent le social comme une totalité, un cosmos, et justifient, sur la base de l'hétérogénéité de telles activités, les relations qu'elles entretiennent mutuellement. On pourrait dans cette mesure comprendre le statut des bovins dans les sociétés est-africaines de manière analogue au statut des porcs dans les sociétés mélanésiennes qu'ont étudiées plusieurs disciples de Dumont.

29 Je me démarquerai cependant de cette analyse sur deux points. La notion dumontienne de hiérarchie, d'une part, se définit exclusivement en fonction de la référence à la totalité cosmique, telle que l'exprime le mythe, et exclut la traduction de ces hiérarchies en termes sociaux. Or, il est clair que la distinction entre Élevage et Agriculture en Afrique se traduit aussi par des hiérarchies sociales: entre hommes et femmes, entre agriculteurs spécialisés et agriculteurs, et même, en Afrique centrale, entre aristocraties pastorales et agriculteurs dominés ${ }^{14}$. En s'intéressant exclusivement, d'autre part, aux modes de clôture transcendantale d'une culture, et non d'une société donnée, Dumont et son école s'interdisent de comprendre les capacités de changement que révèlent les sociétés concrètes, et qui tiennent au fait que l'Élevage, par exemple, qu'il s'agisse de celui des bovins en Afrique ou de celui des porcs en Mélanésie, est certes déterminé par les représentations hiérarchiques que nous avons identifiées, mais reste une activité s'exerçant par l'intermédiaire de pratiques et de savoirs techniques, dans des milieux naturels et humains particuliers, qui sollicitent le changement, inscrivent ces sociétés dans le temps, dans l'histoire.

Ainsi les activités rituelles, que je situe comme Dumont au cœur de la pratique des sociétés « holistes », ne sont pas figées une fois pour toutes dans les représentations qui les commandent; elles sont reformulées constamment en fonction de la réorganisation de ces représentations : les Dinka deviennent des Nuer -et cela a des conséquences décisives sur leur vie matérielle et sociale. Les travaux pionniers de Dumont demandent à être complétés par une analyse préalable des mécanismes qui sont à l'origine de ces représentations. Claude Lévi-Strauss s'est penché sur ce problème dans une bonne partie de son œuvre, qui pose la question de la nature des classifications (1962) ou déconstruit la structure du récit mythique (1964-1971).

31 Je me propose de jeter un pont entre ces deux auteurs, par ailleurs si profondément différents, en introduisant, avec la notion de fétichisme, une possibilité d'analyser simultanément les mécanismes généraux qui président à la constitution des sociétés " holistes ", toutes l'étant à vrai dire, sauf nos sociétés modernes - je suis en accord sur ce point avec Dumont-, et les conditions particulières qui président à la formation comme aux transformations de sociétés particulières. La tâche est ambitieuse, j'en suis conscient, et je ne peux présenter ici que quelques éléments d'analyse, organisés justement autour de la notion de fétichisme du bétail dans les sociétés est-africaines. 
Le fétichisme du bétail et l'ordre social est-africain

32 J'ai développé à plusieurs reprises l'analyse de cette notion de fétichisme et la présentation de son usage pour comprendre le fonctionnement des sociétés où les valeurs du bétail s'inscrivent au centre des représentations et des pratiques sociales ${ }^{15}$. M'inspirant plus particulièrement de Marx et de son idée du fétichisme de la marchandise, j'entends par fétichisme un mécanisme de la pensée qui procède par un déplacement, un transfert des significations attribuées à une activité, ou au produit d'une activité humaine, qui "dénie $»^{16}$ en quelque sorte à celle-ci la faculté de se présenter en tant que telle et en fait la cause initiale des relations de toutes sortes nouées entre les hommes ${ }^{17}$.

Appliquée à l'univers du bétail qui structure les représentations et les pratiques des sociétés est-africaines, cette notion de fétichisme permet d'éviter, me semble-t-il, un double écueil. Le premier consisterait à assigner une réalité d'ordre essentialiste, ontologique, au travail, à l'élevage des animaux domes-tiques en l'occurrence, et à la division du travail dans lequel il s'inscrit, en la distinguant, comme une réalité positive, des représentations que s'en font les intéressés ${ }^{18}$. Le second, qu'illustre la notion de «hiérarchie » telle que l'entend Dumont, serait la prise en compte prioritaire de ces représentations du point de vue de la totalité qu'elles constituent, et qui détermine une fois pour toutes l'efficacité symbolique des pratiques et des savoirs de la société. La notion de fétichisme du bétail, dont nous allons résumer ici les principaux effets sur l'organisation matérielle et sociale permet de prolonger et des préciser les réflexions de Claude Lévi-Strauss sur l'efficacité symbolique, qu'il aborde trop exclusivement, à nos yeux, sur la base des savoirs (classificatoires) et des discours (mythiques) produits par les sociétés concernées, et non à partir des pratiques (rituelles) qui en sont indissociables.

Très généralement définie, la fétichisation du bétail, dans ces sociétés, consiste en ce que le bétail (bovin) apparaît non pas comme le résultat des activités et connaissances d'éleveurs, collectivement organisés, mais comme l'origine de la vie sociale et la cause de ces activités dont nous avons déjà souligné qu'elles conjuguent ces représentations fétichisées et les techniques et connaissances pastorales en les rassemblant sous l'étiquette d'Élevage. Voyons maintenant comment opère ce déplacement de sens, tout d'abord pour organiser les rapports qu'entretiennent entre eux les membres de ces sociétés. En un second temps, nous examinerons la manière dont ces représentations et pratiques gèrent les relations entre les hommes et le surnaturel.

Il est un domaine privilégié de la réflexion anthropologique : la parenté et de l'alliance de mariage. Pour les sociétés est-africaines, la prise en compte des processus de fétichisation du bétail apporte des compléments indispensables aux travaux classiques. On sait que dans certaines de ces sociétés, chez les Nuer en particulier, le mariage est scellé par le transfert d'une quantité importante de bovins de la famille de l'époux à celle de l'épouse. Dans la dernière partie des Structures élémentaires de la parenté (1949), Lévi-Strauss étudie un mariage de ce type, le mariage lobola pratiqué par les populations bantoues du sud de l'Afrique; il interprète ce mouvement du bétail à l'occasion du mariage comme un élément de constitution de la famille, légitimant les droits du père sur ses enfants dans un contexte de filiation unilinéaire, ainsi que comme une manifestation de la loi universelle de l'échange: en circulant en sens opposé des femmes, les bovins jouent un rôle d'équivalent généralisé; un homme se marie grâce aux bovins reçus pour le mariage de sa sœur. 
La théorie a sans doute sa part de vérité, mais n'est-ce pas celle de l'anthropologue? Autrement dit, est-ce bien ainsi que les Nuer et autres populations africaines se représentent réellement les fonctions et les effets d'une telle pratique sociale ? EvansPritchard nous fournit une autre clef pour interpréter la prestation matrimoniale, qui éclaire sur son efficacité symbolique et sociale en la rapprochant de la règle fondamentale d'exogamie clanique et lignagère :

«Les Nuer expriment ces lois d'exogamie en termes de bétail, car là où le bétail peut passer d'un lignage à un autre en vertu de la parenté, les femmes ne peuvent passer par mariage. On ne peut épouser une femme si l'on appartient à un groupe de parents qui reçoivent une partie de la prestation qui doit être versée pour elle. » (Evans-Pritchard 1937 : 211)

L'exogamie est ainsi justifiée par l'idée d'un bétail possédé en commun aux origines, qui interdit le mariage et autorise, à l'inverse, que du bétail circule à l'occasion du mariage entre les groupes partenaires. Chez les Nuer, contrairement à ce qui est en jeu dans le mariage lobola, on ne peut dire qu'un homme reçoit pour se marier les bovins obtenus grâce au mariage de sa sœur, puisque le bétail réuni pour la prestation, et la distribution de celui-ci dans le groupe de la femme, impliquent le groupe dans son entier avec un barème de constitution et de redistribution établi en fonction de la proximité parentale. Par ailleurs, et à partir des mêmes représentations de l'exogamie en termes de bétail, un homme, dans cette société polygynique, ne peut se marier dans un lignage qui a déjà fourni une épouse à lui-même ou à l'un de ses proches parents agnatiques. D'autres travaux anthropologiques mettent en évidence les mêmes représentations et pratiques. Ainsi, chez les Jie d'Uganda :

«Les gens eux-mêmes définissent et expliquent les interdits matrimoniaux en termes de droits réciproques sur des troupeaux qui entreraient en contradiction avec les droits associés aux nouvelles relations qui s'établiraient [par le mariage]. » (Gulliver 1955)

De manière générale, les relations que les anthropologues identifient et analysent dans ces sociétés comme des relations de parenté, pour une part à juste titre car elles gèrent effectivement la reproduction démographique sexuée du groupe, se réfèrent dans les représentations et pratiques locales à l'appropriation initiale, au partage et à la circulation des bovins. Nul mieux que l'anthropologue israélien Uri Almagor n'a identifié et analysé le fait dans toutes ses conséquences à l'occasion de ses travaux sur une population pastorale du sud de l'Éthiopie, les Dassanetch :

« La parenté effective, écrit-il, s'exprime par des droits sur les troupeaux; le nombre de parents effectifs d'un individu, opposés aux parents éloignés ou à ceux simplement désignés par une catégorie de parenté, est constamment redéfini de cette manière, en accord avec des circonstances fluctuantes. » (Almagor 1978 : 198)

Certes, ces considérations n'interdisent pas de décrire et d'analyser en tant que tel le système de parenté. Mais elles ouvrent sur de nouvelles interprétations qui permettent de dépasser le cadre formaliste pour comprendre le fonctionnement concret de la parenté. Suivons encore les travaux d'Almagor qui, à partir d'une fine analyse ethnographique, se résout à classer les relations de parenté chez les Dassanetch dans le contexte plus large de la catégorie des " partenaires pastoraux » (pastoral partners), dont les relations se définissent par les différents modes de circulation du bétail intervenant entre eux.

40 En effet, la filiation est conçue comme l'appropriation d'un troupeau commun aux origines ancestrales, et le mariage comme la conséquence de la circulation du bétail 
entre des groupes exogamiques; il existe de multiples manières d'établir entre les hommes, dans des contextes rituels ou à leur seule initiative, des relations lil metch qui sont concrètement fondées sur la circulation des bovins. Dès la naissance, le père noue des relations de ce type en faveur de son fils, auprès d'un homme de sa propre génération mais d'un clan différent, une sorte de relation de "parrainage ", si l'on peut s'exprimer ainsi sans céder à trop d'ethnocentrisme. Au moment de la circoncision de son fils, il fera de même, toujours avec un homme de sa génération, mais cette fois de son propre clan. En outre, un homme marié établit, à la naissance de son premier fils, des relations de partenaire pastoral avec quelqu'un dont il donnera le nom à cet enfant. Recouvrant les relations consanguines et affinales, ces liens lil metch tissent progressivement un réseau de rapports contractuels qui déterminent le comportement social de celui qui les a noués, créent des solidarités, étendent les interdits matrimoniaux, orientent le choix des alliances de mariage, etc. Ce réseau se transmet pour une part aux enfants qui l'enrichissent de leurs propres choix relationnels.

Les enjeux sont-ils "matériels", autrement dit techno-économiques dans notre langage contemporain? Sans négliger les effets, qui vont en ce sens, des relations lil metch, contribuant à la construction du troupeau familial et créant une sorte d'« assurance " pour un éleveur dont le troupeau serait frappé par une épidémie ou par la sécheresse, il est clair qu'elles ne sont pas constituées à cette fin par les intéressés. Il s'agit d'ailleurs moins de transferts réels d'animaux que de droits potentiels, qui ne sont activés qu'en certaines circonstances. On notera en outre que la prestation matrimoniale elle-même est le plus souvent transférée très progressivement, sans que cela ne remette en question les droits afférents. Ainsi, toujours chez les Dassanetch, elle n'est remise que bien après le mariage, au moment où le chef de famille, à la suite d'un rite appelé dimi, se retire de la vie active d'Éleveur pour se consacrer au rôle de médiateur politique et de spécialiste rituel.

En définitive, cette circulation des bovins, qui reste souvent virtuelle, constitue moins, selon l'heureuse expression d'Almagor, l'objectif immédiat d'une « capitalisation » du bétail aux mains des éleveurs que la "trame invisible» de la société qui définit les relations entre ses membres; une trame employée pour que chacun réalise son statut d'« Éleveur ", c'est-à-dire les valeurs dominantes de la société, se traduisant en retour par une autorité politique et rituelle qu'incarneront en définitive non pas ceux qui apparaissent comme les "propriétaires » immédiats du bétail, mais ceux qui en maîtrisent l'usage social.

Les Dassanetch, qui ont usé avec le maximum d'efficacité des droits virtuels qu'ils détiennent sur des bovins, ceux qu'ils ont accumulés à travers les relations lil metch, entre " partenaires pastoraux », en tireront, selon l'expression d'Almagor, un « crédit » qu'ils traduiront en autorité sociale. Ce crédit ne peut se manifester qu'au moment où le troupeau familial qu'ils ont constitué, et entretenu par leurs activités matérielles et sociales, se disperse significativement au gré des échanges et de sa transmission aux enfants. Si un Dassanetch tente d'utiliser les animaux qu'il contrôle à des fins personnelles, pour accroître son troupeau et renforcer ses richesses, il risque la grave accusation de forgoginte, une sorte d'ostracisme qui le bannit de la vie sociale, politique et rituelle. Almagor conclut ainsi :

«Le crédit social d'un homme ne provient pas tant des biens qu'il possède réellement [le nombre de têtes de bétail] que de sa capacité à manipuler des relations sociales et à agir comme médiateur pour accroître son crédit social qui lui permettra en retour de réaliser plus aisément ses propres intérêts. » (1978:131) 
Sans évoquer la notion de fétichisme du bétail, la magistrale description ethnographique de la société " pastorale » dassanetch à laquelle procède Almagor, en illustre certains des effets les plus manifestes. Les relations sociales sont généralement fondées sur la circulation du bétail au sein de la société dassanetch; cette circulation a pour effet d'assurer parallèlement la constitution des troupeaux et des familles -une constante qu'ont soulignée de nombreux auteurs dans ces sociétés pastorales estafricaines-, de répartir entre celles-ci les moyens de production, de fournir le cadre de la division du travail, de satisfaire les besoins, alimentaires et autres, des unités de production. Mais ce n'est pas en ces termes que les Dassanetch conçoivent leur société : ces relations sociales ne sont que l'expression, temporelle et changeante, de la « trame invisible » qui lie les hommes et leurs vaches depuis le temps primordial dont rendent compte les mythes des origines, à travers d'intenses pratiques rituelles. Cette trame, invisible mais bien présente dans les représentations et pratiques des Éleveurs, ne prend elle-même sens qu'en se référant à celle qui unit plus clairement encore, depuis les origines, les hommes et le surnaturel par l'intermédiaire du bétail.

Le rituel sacrificiel

Les mythes d'origine soulignent la place tenue par l'idée de sacrifice dans la constitution de l'univers symbolique du bétail. Donné par Dieu, le bétail est destiné à lui revenir au moyen du sacrifice. Selon les Masai par exemple, c'est parce que les femmes ont fait un mauvais usage du bétail ou des animaux sauvages qu'elles possédaient, en les mettant à mort pour des raisons séculières et utilitaires, que la possession en revient aux hommes qui ont la permission divine de les employer pour des sacrifices, ces derniers apparaissant comme la finalité ultime de la possession des bovins, de la pratique de l'Élevage. Evans-Pritchard rend compte de cette idée dans un texte bien connu :

« Un Nuer ne considère pas son bétail comme le fait un éleveur [occidental], pour la viande ou le lait qu'il fournit. Sa relation avec les animaux est rendue plus complexe, en dehors de leur utilisation pour le mariage, par le fait qu'ils sont destinés à être sacrifiés. Ce n'est pas simplement qu'il ne doit pas tuer une tête de bétail en dehors du sacrifice, parce que, s'il utilisait ainsi son bétail, il disposerait de moins de ressources pour se nourrir, se marier ou pour les rituels. Ce n'est pas seulement une injonction négative. Ce n'est pas "Tu ne dois pas tuer", mais : “Tu dois sacrifier". Ce n'est pas qu'ils ne puissent tuer que pour sacrifier, mais plutôt qu'ils doivent sacrifier pour tuer. » $(1956: 269)$

Ces remarques d'Evans-Pritchard peuvent s'appliquer de manière générale aux éleveurs est-africains et, notons-le au passage, à nombre de sociétés qui pratiquent l'élevage d'animaux domestiques. Les bovins sont destinés, prédestinés serait mieux dire encore, à être sacrifiés. En contrepoint, la victime du sacrifice sera appelée bœuf ou vache, s'agirait-il d'un concombre que les Nuer «sacrifient " quand ils ne disposent pas de bétail ${ }^{19}$. Cette prédestination sacrificielle des bovins rappelle que s'ils sont associés initialement au surnaturel et permettent de restaurer lors de chaque sacrifice la relation entre les hommes et la divinité qui les leur a attribués, ce sont aussi des initiatives humaines, décidant du temps et du lieu des rituels, qui font passer la nature et la société de l'indifférenciation primordiale à leur état actuel. La répétition de l'acte sacrificiel, conçu comme la finalité de l'Élevage, a vocation à évoquer l'ordre cosmique aux différents moments de sa constitution. À l'initiative des hommes, le sacrifice rétablit d'abord la relation originelle instaurée entre les hommes et le surnaturel par l'intermédiaire des bovins : 
« Il [le bétail] est le moyen par lequel les hommes entrent en communication avec Dieu [...] Il est [...] le lien entre le perceptible et le transcendantal. En remplissant ce rôle, le bétail protège un homme et sa famille du malheur, et il le conçoit ainsi dans son ensemble comme un troupeau qui, depuis les origines, a aidé ses ancêtres en difficultés, remplissant à chaque génération les mêmes services sacrificiels. » (Evans-Pritchard $1956: 271$ ) des hommes. En même temps que la capacité de sacrifier légitime l'attribution du bétail à ceux qui se consacrent à l'Élevage -ainsi les femmes ne sacrifient-elles pas-, l'exercice programmé des rituels sacrificiels est, chez les Nuer, l'ultime raison du regroupement de ceux qui sont liés par une filiation commune : Verdon nous a rappelé que la cause dernière de la constitution des lignages et des clans dans la société nuer est la réalisation de ces rituels sacrificiels. Effectuer ces rituels en des circonstances régulières, reproduisant l'organisation lignagère de la société (Nuer) ou celle des classes d'âge (Masai), ou encore en des circonstances exceptionnelles, en cas de mort, de maladie, de sécheresse, etc., restaure l'ordre social et naturel et rend compte de l'efficacité symbolique des bovins telle qu'elle se manifeste dans le cadre du fétichisme du bétail.

Pour conclure

48 Au bétail est ainsi attribuée la propriété d'être à l'origine des rapports sociaux; il constitue la trame invisible qui tisse les relations entre les hommes et entre ceux-ci et le surnaturel, inscrivant " société » et " nature » dans l'ordre cosmique où ces notions perdent les significations que nous leur attribuons dans notre propre système de pensée. Ce que nous avons appelé le fétichisme du bétail n'est pas la manifestation d'une vision imaginaire, illusoire ou fantas-matique, et son efficacité, certes d'ordre symbolique, détermine réellement, matériellement, la manière dont les Éleveurs organisent leurs activités pastorales à travers un ensemble de techniques et de savoirs, et gèrent aussi leurs relations sociales.

La technologie comparée ne peut se construire sur la seule base des représentations que nous nous faisons dans nos sociétés de ce qui est technique, action matérielle finalisée en tant que telle, et de ce qui ne l'est pas, en distinguant activités rituelles et techniques par exemple (Bonte 1999) là où elles apparaissent intimement liées dans l'observation. Dans cette perspective, les représentations qui, sous forme de mythes, président dans nombre de sociétés à la définition de l'activité technique ne sont pas seulement des «mythes d'origine ", comme on les interprète souvent. Ils fournissent les clefs de l'efficacité symbolique de ces activités humaines que sont les activités techniques, fondant leur efficacité matérielle. Les mythes d'origine du bétail dans les sociétés est-africaines apportent en définitive un éclairage irremplaçable, non seulement sur la nature des activités pastorales proprement dite, mais sur la manière dont ces sociétés conçoivent et organisent leur vie sociale. 


\section{BIBLIOGRAPHIE}

Almagor, Uri

1978. Pastoral Partners. Affinity and Bond Partnership among the Dassanetch of South West Ethiopia. Manchester : Manchester University Press.

Becquemont, Daniel \& Pierre Bonte

2004. Le Travail nommé. Mythologies du travail. Paris : L'Harmattan.

s/presse L'illusion de l'échange. Paris : L'Harmattan.

Bonte, Pierre,

1981. "Ecological and economic factors in the determination of pastoral specialisation ", Journal of Asian and African Studies XVI, 1-2 : 33-49.

1984. « Le bétail produit les hommes. Sacrifice, valeur et fétichisme du bétail en Afrique de l'Est », Uomo IX 1-2 : 121-147.

1985. «Faits techniques et valeurs sociales : quelques directions de recherche », Techniques \& culture $5: 19-48$.

1991. « To increase cows, God created the kings. The function of cattle in interlacustrine societies ", pp. 62-86, in D. Galaty et P. Bonte (eds), Herders, Warriors and Traders. Pastoralism in Africa. Boulder/San Francisco, Oxford/Westview Point.

1999. «Travail, techniques et valeur. Contributions (nouvelles) au débat sur rites et techniques », pp. 15-30, in J.-L. Jamard, A. Montigny et F. R. Picon (eds), Dans le sillage des techniques. Hommages à Robert Cresswell. Paris : L'Harmattan.

Burton, John-W.

1980. « Class of Atuot religious thought ", Journal of Religion in Africa XI (2) : 66-105.

1981. "The wave is my mother husband : a pictorial theme in pastoral nilotic ethnology », Cahiers d'Études africaines 21 (4) : 459.

Cartier, Michel (ed.)

1983. Le Travail et ses représentations. Paris : Éditions des Archives contemporaines.

Chang, Cynthia

1982. « Nomads without cattle. East African foragers in historical perspectives », pp. 269-82, in E. Leacock and R. Lee (eds), Politics and History in Band Societies. Cambridge/Paris : Cambridge University Press/Éditions de la Maison des Sciences de l'Homme.

Dumont, Louis

1983. Essai sur l'individualisme moderne. Paris : Le Seuil.

Evans-Pritchard, Edward E.

1937. « Economic life of Nuer (part one) », Sudan Notes and Records 20 : 209-46.

1956. Nuer Religion. Oxford : Clarendon Press.

1968. Les Nuer. Description des modes de vie et des institutions politiques d'un peuple nilote. Paris : Gallimard (1ère édition 1940).

Evens, T.M.S.

1978. « Leopard skins ans paper tigers : "choice" and "social structure" among the Nuer ", Man 13 (1) : 100-115. 
Galaty, John

1977. In the pastoral image : The dialectic of Masai identity. PhD, University of Chicago.

Galaty, John \& Pierre Bonte

1991. Herders, Warriors and Traders. Pastoralism in Africa. Boulder/San Francisco/Oxford :Westview Point.

Godelier, Maurice

1984. L’Idéel et le matériel. Pensée, économies, sociétés. Paris : Fayard.

Grevel, Peter J.

1971. «The leopard skin chief : an examination of political power among the Nuer », American Anthropologist 73 (5) : 115-120.

Gulliver, Philipp

1955. The Family Herds. London : Routledge and Kegan.

Herskovits, Melville J.

1926. « The cattle complex in East Africa », American Anthropologist $28: 230-272,361-388,494-528$, 633-664.

Kelly, Raymond C.

1985. The Nuer Conquest. The Structure and the Development of an Expansionnist System. Ann Arbor: The University of Michigan Press.

Lévi-Strauss, Claude

1949. Les Structures élémentaires de la parenté. Paris/La Haye : Mouton.

1962. La Pensée sauvage. Paris : Plon.

1964-71. Mythologiques (4 volumes). Paris : Plon.

Merker, M.

1971. The Masai. Ethnographic Monography of an East African Semitic People : Berlin : Dietrich Reiner (traduction anglaise, Human Relations Area Files) (1ère édition 1910).

Pouillon, Jean

1975. Fétiches sans fétichisme. Paris : François Maspéro.

Rigby, Peter

1971. « The symbolic role of cattle in Gogo ritual », pp. 257-292, in T.O. Beidelman (ed.), The

Translations of Culture. London : Tavistock.

Sahlins, Marshall

1961. «The segmentary lineage : an organisation of predatory expansion », American

Anthropologist 63 : 322-345.

Southall, Adam

1985. « The illusion of Nath agnation », Ethnology 24 (1) : 1-20.

Spencer, Paul

1973. Nomads in Alliance. Symbiosis and Growth among the Rendille and the Samburu of Kenya. London : Oxford University Press.

Verdon, Michel

1984. "Where have all their lineages gone? Cattle and descent among the Nuer », American Anthropologist $3:$ 566-579. 


\section{NOTES}

1. Elles ne distinguent pas en particulier, comme dans les zones plus septentrionales de l'Afrique, des populations qui pratiquent l'élevage dans le contexte d'un pastoralisme nomade et d'autres populations agro-pastorales : les Masai ne sont pas des nomades, bien qu'éleveurs spécialisés.

2. Nous emphatisons le terme par cette majuscule, comme nous l'avons fait dès le titre de l'article.

3. Les éleveurs possèdent aussi du petit bétail, des moutons et des chèvres, mais n'ont pas, à son égard, la même attitude que vis-à-vis de leurs vaches, bœufs et taureaux.

4. Le lecteur comprendra aisément, et nous y reviendrons de manière plus théorique, que nous ne confondons pas l'activité technique avec sa dimension matérielle.

5. Ce récit, comme d'autres cités par la suite, est emprunté à l'ouvrage d'un auteur allemand du début du XXe siècle, M. Merker (1910). Précisons immédiatement que l'intérêt du livre, qui tient à la période où ont été recueillis ces mythes, doit être nuancé en fonction de l'idéologie de l'auteur : il pense identifier dans les sociétés pastorales est-africaines, en se fondant sur l'idée répandue d'une puissance créatrice originelle dans leurs idées religieuses, les traces d'influences sémites qui le conduisent à une relecture inspirée des mono-théismes de ces mythes d'origine.

6. La technique de prélèvement du sang des animaux vivants, à des fins alimentaires, en utilisant un petit arc et une flèche pour percer la veine jugulaire, est de fait assez généralement répandue en Afrique de l'Est.

7. La relecture chrétienne des mythes masai par Merker est ici assez évidente mais, rapprochée d'autres recueils de mythes ultérieurs, elle ne remet pas en question la signification première de ceux-ci.

8. La propriété des bovins est exclusivement masculine, même si les femmes, chez les Masai, ont l'usufruit (le lait) des têtes de bétail que leur confie leur mari.

9. On pense ainsi qu'une partie de la société nuer actuelle est constituée de groupes dinka qui ont été absorbés dans la société nuer en « expansion ». Cette analyse a été plus particulièrement développée par R.C. Kelly (1985).

10. Sur les fonctions de ce personnage dans la société nuer, on pourra consulter, outre les travaux d'Evans-Pritchard, les articles de Grevel (1971) et d'Evens (1978).

11. C'est le cas notamment de certains pasteurs masai qui pratiquent l'élevage dans des régions où les conditions climatiques, la pluviométrie en particulier, permettraient la pratique simultanée de l'agriculture (Bonte 1981)

12. On pourra consulter les travaux de Chang sur les Torrobo (1982). Il est vraisemblable que des mouvements réguliers de population se produisaient entre Masai et Torrobo. Ceux-ci peuvent acquérir du bétail et se "pastoraliser ", se transformant ainsi en Masai. À l'inverse, des Masai qui perdent leurs troupeaux pouvaient rejoindre les Torrobo, pratiquer comme eux la chasse et la cueillette, et devenir, de ce fait, des Torrobo (Spencer 1973).

13. Faiseurs de médecines, de pluie.

14. Hiérarchies qui sont au point de départ des événements tragiques qu'a connus le Centre de l'Afrique ces dernières années, au Rwanda et au Burundi en particulier. J'ai développé ce point ailleurs (Bonte 1991).

15. En particulier dans un premier article paru il y a une vingtaine d'années (1984). Dans un ouvrage récent (Becquemont \& Bonte 2004), nous effectuons une synthèse de ces travaux et de l'usage anthropologique (comparatif) de cette notion de fétichisme. 
Rappelons qu'elle a d'abord été engagée théoriquement par Auguste Comte avant d'être utilisée par Karl Marx pour décrire le fétichisme de la marchandise dans l'« Introduction » au Capital, et d'être reprise dans un autre contexte par Sigmund Freud. Longtemps délaissé et remis en question, c'est récemment que le terme a été à nouveau employé par des anthropologues : en particulier par Jean Pouillon (1975), auquel je me permets de rendre hommage, tout en suggérant de renverser la formule qu'il énonce " fétiches sans fétichisme ", par la formule inverse, " fétichisme sans fétiches », qui me semble mieux correspondre au statut de cette notion dans les sciences sociales

16. Je préfère cette terminologie à celle d'aliénation qu'emploie souvent Marx, et qui peut impliquer une perte de significations par rapport à une réalité première, essentielle, positive. Elle jette par ailleurs un pont vers l'analyse freudienne qui considère le fétichisme comme l'effet du déni de l'absence de phallus féminin, manifestation pathologique mais aussi expression très générale de l'interrogation sur la distinction des sexes.

17. Le fétichisme de la marchandise se manifeste ainsi par le fait que les relations d'échange entre les hommes dans la société capitaliste apparaissent comme l'effet de relations entre les choses : les marchandises qui circulent dans l'échange.

18. C'est l'idée qui transparait à travers l'expression « le travail et ses représentations » et qui a inspiré un ouvrage collectif par ailleurs d'un grand intérêt (Cartier 1983) ou encore, dans la distinction opérée par Maurice Godelier entre la part matérielle et la part idéelle du réel (1984).

19. Il en est de même chez leurs voisins atuot : « Dans le contexte spécifique du sacrifice, toute victime est désignée sous le nom de yang decan, la vache du Créateur. » (Burton 1980 : 102).

\section{RÉSUMÉS}

E. E. Evans-Pritchard définissait les Nuer comme un "peuple du bétail» (cattle people). Developpant cette notation, on montre ici que dans un certain nombre de populations d'Afrique de l'Est, le bétail se voit attribuer dans les mythes la propriété d'être à l'origine des rapports sociaux. Il constitue la trame invisible qui tisse les relations entre les hommes et entre ceux-ci et le surnaturel, à travers les rituels sacrificiels en particulier. Ce que nous appellons fétichisme du bétail, en attribuant une portée plus générale à cette notion de fétichisme, n'est pas la manisfestation d'une vision illusoire et fantasmatique. Son efficacité, certes d'ordre symbolique, détermine réellement, matériellement, la manière dont les éleveurs organisent leurs activités pastorales à travers un ensemble de techniques et de savoirs.

\section{Cattle people. Mythical origins and ritual practices in East Africa}

E. Evans Pritchard has defined the Nuer as a «cattle people ». Developing this idea, we can say that in several East-african populations, the cattle is seen to be at the origin of social relations, weaving the invisible frame of relations between men and between men and the supernatural, specially through sacrificial rituals. What we call «cattle fetichism»-without restricting the meaning of this notion- is not an illusory and fantomatic vision. The efficiency of fetichism is of 
course on a symbolical level, but it really materially determines, the way cattle farmers organize their pastoral activities, through a set of techniques and knowledges

Los «pueblos ganaderos ». Orígenes míticos y prácticas rituales del pastoreo en Africa Oriental

E. E. Evans-Pritchard solía definir a los Nuer como un "pueblo ganadero" (cattle people). Desarrollaremos esta cuestión, para mostrar cómo en los mitos de un cierto número de poblaciones de Africa Oriental, se atribuye al ganado la propiedad de ser el origen de las relaciones sociales. Es la trama invisible que teje las relaciones entre los hombres, y entre estos y lo sobrenatural, en particular a través de los ritos de sacrificio. Aquello que denominamos fetichismo del ganado, atribuyendo una acepción más general a este concepto de fetichismo, no es la manifestación de una visión ilusoria o fantasmal. Su eficacia, naturalmente dentro del orden simbólico, determina realmente, materialmente, la manera en la que los criadores de ganado organizan sus actividades de pastoreo a través de un conjunto de técnicas y saberes.

\section{INDEX}

Mots-clés : rites, Afrique de l'est, bétail, fétichisme, mythes, sacrifice

Keywords : East Africa, Cattle, myths, rituals, sacrifice, fetichism

\section{AUTEUR}

\section{PIERRE BONTE}

CNRS, LAS, 52 rue du Cardinal Lemoine, 75005 Paris 